\title{
Letter to the Editor for 'Current Addiction Reports'-Game Transfer Phenomena and Dissociation: a Reply to Guglielmucci et al. (2019)
}

\author{
Angelica B. Ortiz de Gortari ${ }^{1,2} \cdot$ Mark D. Griffiths $^{3}$
}

Published online: 1 May 2019

(C) The Author(s) 2019

To the Editor,

It was with great interest that we read Guglielmucci et al.'s [6] paper 'Dissociation in Problematic Gaming: a Systematic Review' in Current Addiction Reports. Their systematic review of empirical studies examined associations between problematic gaming and phenomena considered to be on the continuum between normal and pathological forms of dissociation. Literature with this focus is scarce and we thank the authors for their contribution. However, the purpose of this letter is to discuss the authors' conceptualisation of our research on Game Transfer Phenomena (GTP) as dissociative phenomena.

... excessive video game use is linked to a variety of dissociative phenomena (e.g. depersonalisation experiences, escapism, psychotic-like experiences, game transfer phenomena) (p.1).

Guglielmucci et al.'s conceptualization of our research on GTP was too simplistic. Game Transfer Phenomena are both more detailed and more nuanced. GTP are involuntary phenomena that comprise sensory, perceptual, cognitive, and self-agency transient changes or intrusions in direct relationship to (i) videogame content/features, (ii)

Angelica B. Ortiz de Gortari

angelica@gametransferphenomena.com

Mark D. Griffiths

mark.griffiths@ntu.ac.uk

1 Psychology and Neuroscience of Cognition Research Unit, University of Liège, Quartier Agora Place des Orateurs, 3B33 4000, Liège, Belgium

2 The Centre for the Science of Learning and Technology, University of Bergen, Christiesgate 13, Vektergården N-5020, Bergen, Norway

3 International Gaming Research Unit, Psychology Department, Nottingham Trent University, 50 Shakespeare Street, Nottingham NG1 4FQ, UK subjective phenomena experienced while playing (e.g. immersion, embodiment, telepresence), and (iii) gamerelated hardware or peripherals [9]. Specific manifestations include perceptual distortions (distortions of objects, environments, sounds, body, or time), pseudohallucinations (e.g. images overlaying game-related objects or sounds coming from objects associated with the game), imagery (e.g. imagining game elements), sensations of unreality and disembodiment (e.g. out-of-bodylike sensations and feelings), automatic mental processes (e.g. source monitoring errors, attentional bias), and involuntary actions/behaviours [11]. GTP connote the interplay of physiological, perceptual, and cognitive mechanisms and happen suddenly and outside the gamers' control [18], although some gamers claim to be capable of controlling their GTP visualisations after a while [13].

The inclusion of GTP as dissociative phenomena by Guglielmucci et al. [6] raises interesting questions regarding the nature of GTP. For instance, should GTP be considered dissociative in the continuum from episodic intrusions with game content (e.g. images, sounds, thoughts, urges, impulses) that can awaken feelings and sensations of unreality, to engrossment in mental actions (e.g. replays of the game) or multisensory sensations and automatic actions toward gamerelated stimuli that lead to absentmindedness? Based on the broad and rather general definition of dissociative disorders according to DSM-5 [1] used by Guglielmucci et al., to describe 'dissociations', the intrusive nature of GTP could be arguably be considered 'dissociative':

Dissociative disorders are characterised by "disruption of and/or discontinuity in the normal integration of consciences, memory, identity, emotion, perception, body representation, motor control, and behaviour" ([1], p. 291).

We acknowledge there are phenomenological similarities between GTP and diagnostic features of dissociative disorders. However, we advise caution in generalisations which 
may lead to overestimating the impact of GTP on gamers' lives at this early stage in the study of GTP. This is because independently, the form of dissociation (normative or pathological) involves absentmindedness and implies different degrees concerning the sense of experimental disconnectedness with the self and with the environment [3].

Initial observations suggest there are associations between failures in cognitive control (e.g. attention, inhibitory control), inhibitory control failures, and GTP $[15,17 \bullet \bullet]$. Mix-ups and confusion between game elements and physical stimuli that often resemble game content have also been observed [10, 12]. However, suspension of reality and/or selfconsciousness only occur in some forms of GTP and in specific circumstances. Not all forms of GTP involve absentmindedness or appear to disrupt ongoing behaviours. Still, since intrusions with game content (e.g. hearing music from the game) are products of 'a living game experience' and are usually associated with events in the game, every intrusion can potentially awaken memories, feelings, false expectations, urges, impulses, and (in more extreme cases) sensations to be in the game. The degree to which game intrusions and changes in perception can be disruptive and lead to actions without awareness requires further empirical investigation.

Another significant aspect of many forms of GTP is the presence of game-related stimuli as a trigger to subsequent behaviour. Consequently, GTP that occur in fully awake states when not playing the game manifest in parallel or as complementary to ongoing thoughts and behaviours and do not result in zoning out. For example, experiencing an inner voice of a command from within a videogame (e.g. 'Go, Go, Go', a voice command from the game Team Fortress) while wanting individuals to move faster as they board a subway train [14]. However, we acknowledge that attentional bias and hyperfocus toward game-related stimuli or memories/ feelings from a videogame may come at a cost and potentially lead to absentmindedness. Moreover, GTP are not the result of volitional acts of avoiding reality as escapism; GTP can occur suddenly as a homeostatic mechanism when social situations provoke anxiety, as well as by sleep deprivation and arousal $[12,13]$.

When examining dissociation in GTP, some forms of GTP are dissociative per se, and dissociative instances have been identified in gamers' self-reports [12-15] and when using the GTP Scale [21]. We agree with Guglielmucci et al. that the forms of GTP they included in their paper involved dissociative phenomena or were intimately related because they involved altered body perceptions, including altered perception of time:

sensations of derealisation and depersonalisation triggered by external cues associated with the video game", "mind disconnected from the body", "feeling the body as different after playing a video game", "feeling body movement after playing", "feeling tactile sensations ([6], p. 8).

However, some of the altered perceptions provoked by the virtual immersion identified in previous GTP studies are highly prevalent and it appears problematic to classify them as dissociative rather than as simple neural adaptative phenomena, even though some of them are intrinsically related to dissociative phenomena such as vestibular adaptations to autoscopy $[2$, 18]. An example of this is "feeling body movement after playing" (i.e. whole body self-motion such as feeling the illusion of movement from the game) [18], which is one of the most common body-related phenomena reported (47-51\%) among studies with general games $[4 \bullet \bullet, 17 \bullet \cdot]$. This mainly happens when trying to fall asleep and appears to be explained by vestibular neural adaptations similar to the Mal de Debarquement Syndrome [8]. Another example of altered perceptions frequently reported in GTP studies is when objects are perceived as levitating or expanding, which typically happens after gamers look away from the screen when playing dance/music games [12]. This appears to be explained by motion aftereffects of a waterfall type $[5,18]$. Moreover, it is well-known that immersion in virtual reality (VR) when individuals are wearing VR headsets is capable of overriding top-down knowledge with bottom-up perceptual mechanisms giving rise to illusions of transfer of body ownership [23]. Even acute dissociative symptomatology can be induced via VR immersion [24].

A broader examination of GTP by Ortiz de Gortari and Larøi [19] includes additional and redefined items that assess altered body-related phenomena and relate dissociative phenomena: (i) out of body/autoscopy (i.e. perceiving the self in third-person perspective as in a videogame), (ii) derealisation, (iii) depersonalisation, (iv) stupor-like phenomena (i.e. remaining immobile, unable to articulate words or being verbally unresponsive due to being stuck in the mindset of a videogame), (v) sensation of ownership of virtual limbs, (vi) perceiving changes in the characteristics of one's own body (e.g. sizes, heaviness), (vii) involuntary movements of limbs (toward a game-related stimuli or jerk or twitch), and (viii) mimicking game characters (e.g. postures, gestures). Consequently, a classification of dissociations manifesting in GTP is as follows:

- Sensory/perceptual neural adaptations and other physical aftereffects (e.g. visual distortions, loss of visual acuity, uncoordinated movements, vertigo) most probably heightened by prolonged gaming sessions and mental fatigue, interpreted as still being in the game or being the game character usually soon after playing.

"I was playing ... about four hours straight. When I stood up, I had a massive head rush. I thought I was a Jedi in a cave for about five seconds. I was worried that 
the giant birds in the game's caves were going to attack me. I was confused and afraid” ([18], p. 111).

- Automatic responses toward game-related stimuli either sensory/perceptual intrusions of game elements, usually manifesting externally, (e.g. seeing images in the periphery, hearing sounds coming from objects or nowhere) or misinterpretation and/or confusion of objects and/or events associated with the game that have become conditioned stimuli capable of eliciting conditioned responses (impulsive and mainly emotional responses), which subsequently can end in dissociative reactions such as sensations and/or feelings of being back in the game and/or impulsive acts without awareness toward the gamerelated stimuli.

"After days of playing ... I was at the gas station. There were two cars by each other, and I thought there would be enough room to squeeze through. I got all the way up to the cars and then realized that this was real life" ([15], p. 444).

- Engrossment in automatic mental actions with gamerelated stimuli characterised by replaying the game in one's mind in a stereotypical manner (e.g. looking for patterns, scanning for game objects, applying game strategies) that can lead to episodes of absentmindedness. In more extreme cases - typically after ceasing playthoughts appeared and lowered cognitive flexibility to switch from virtual to real life tasks as in perseverative mental states [15].

\section{"I played ... and got all the hidden packages at once.} When I quit playing, I was looking in the corners of the rooms for hidden packages. It was really odd" ([15], p. 439).

- Engrossment in vivid imagery of the game, which beyond thoughts and imagination of the game, also occurs when gamers experience visual sensations of relatively prolonged duration when they close their eyes and feel like they are replaying the game (i.e. closed-eye hallucinations, sometimes even accompanied with sound). This tends to occur not only in the liminal state between wakefulness and sleep (i.e. hypnagogia) but also during periods of wakefulness (i.e. parahypnagogia) [7], which can lead to episodes of absentmindedness.

"I don't usually play it in the evening now ... When I go to bed, I can see Tetris shapes on the back of my eyelids, and I try to make the shapes all fit together... It's sort of fun for a while but then I think "I need to sleep!" '([13], p. 100).
Guglielmucci et al. noted the potential association between the video-terminal dissociative trance (VTDT) and the "translation of gaming experience to real life" (p. 10). The VTDT was proposed by Schimmenti and Caretti [22] as a clinical construct "characterized by clusters of symptoms in the psychological domains of addiction, regression, and dissociation in the individual's interaction with the computer and its applications" (p.64). According to the authors, the VTDT "may involve significant disturbances in the states of consciousness, identity, and memory, the dilution of self-awareness and self-integrity, and the replacement of the customary sense of personal identity by a new virtual identity" (p. 64). Regarding the transfer of experience to real life, Guglielmucci et al. went on to say:

In their study, Ortiz de Gortari and Griffiths [17••] found that a high prevalence of gamers experienced GTP at some point. This might be consistent with the video-terminal dissociative trance hypothesis, which predicts that an alteration of mental and behavioural functioning may occur in the individual at extreme levels of absorption into the game, due to an alteration in cognitive and affective processing of information linked to the excessive gaming and a consequent translation of gaming experience to real life ([6], p. 10).

Our research has showed that there is a significant relationship between GTP, immersion, and playing to escape from the real world $[10,16]$. More specifically, engaging in activities that involve focusing the attention on specific game elements by exploring and customising appear to be relevant for GTP [16]. Regarding problematic and excessive playing, GTP are correlated with fulfilment of gaming disorder criteria [19]. Frequency of playing and session length predicts severe GTP (i.e. experiencing GTP many times and/or experiencing two or more types of GTP) [20••]. However, gaming habits are not always consistent because most gamers experience mild levels of GTP $[19,20 \bullet$. For instance, session length in different dimensions of GTP only shows significant differences in altered body perceptions and behaviours (e.g. verbal outbursts, involuntary movement of limbs), but not visual perceptions, auditory perceptions, or thought-related experiences.

In summary, a more in-depth understanding is needed concerning the relationship between dissociations in videogame playing and problematic gaming. This can only emerge from research that examines underlying mechanisms (e.g. physiological, behavioural) involved in dissociative trance-like states (during playing) and dissociations manifesting after playing, instead of only focusing on the relationship between dissociative disorders/traits and gaming and problematic gaming. It is important to bear in mind that GTP are almost ubiquitous among gamers $(81-97 \%$; $N>6000 ; 15-$ 60 years old) (Dindar \& $[10,17 \bullet \bullet, 19])$. GTP are mostly appraised as positive by gamers $[17 \bullet \bullet]$. Most gamers who report 
GTP do not have any mental disorder nor were they under the influence of psychoactive substances when they experienced GTP $[10,16]$. The most common forms of GTP do not appear to affect ongoing behaviours or lead to sensation of unreality of self and body [11]. However, when GTP become severe (i.e. several forms and frequently), 58\% reported distress and/ or dysfunction in one study [20••]. The impact of GTP appears to be related to how sensory/perceptual changes or intrusions are interpreted and appraised, what subsequent behaviour they lead to, and under what circumstances they manifest. Since most GTP manifest in diurnal contexts, it is crucial to evaluate when GTP can genuinely affect normal functioning (e.g. disrupt task performance, and in extreme cases, lead to potential accidents) and psychological health (e.g. from awkward moments to questioning self-identity and mental stability).

Open Access This article is distributed under the terms of the Creative Commons Attribution 4.0 International License (http:// creativecommons.org/licenses/by/4.0/), which permits unrestricted use, distribution, and reproduction in any medium, provided you give appropriate credit to the original author(s) and the source, provide a link to the Creative Commons license, and indicate if changes were made.

\section{References}

Papers of particular interest, published recently, have been highlighted as:

•- Of major importance

1. American Psychiatric Association. Diagnostic and statistical manual of mental disorders. 5th ed. Arlington, VA: American Psychiatric Publishing; 2013.

2. Blanke O, Mohr C. Out-of-body experience, heautoscopy, and autoscopic hallucination of neurological origin: implications for neurocognitive mechanisms of corporeal awareness and self-consciousness. Brain Res Rev. 2005;50(1):184-99.

3. Cardeña E, Carlson E. Acute stress disorder revisited. Annu Rev Clin Psychol. 2011;7:245-67.

4.• Dindar M, Ortiz de Gortari AB. Turkish validation of the Game Transfer Phenomena Scale (GTPS): measuring altered perceptions, automatic mental processes and actions and behaviours associated with playing video games. Telematics Inform. 2017;34(8):1802-13 This study corroborated the original validation of the Game Transfer Phenomena Scale by Ortiz de Gotari, Pontes and Griffiths (2015).

5. Dyson BJ. She's a waterfall: motion aftereffect and perceptual design in video games involving virtual musicianship. Perception. 2010;39(1):131-2.

6. Guglielmucci F, Monti M, Franzoi IG, Santoro G, Granieri A, Billieux J, et al. Dissociation in problematic gaming: a systematic review. Curr Addict Rep. 2019. https://doi.org/10.1007/s40429019-0237-z.

7. Gurstelle EB, de Oliveira JL. Daytime parahypnagogia: a state of consciousness that occurs when we almost fall asleep. Med Hypotheses. 2004;62(2):166-8.

8. Ombergen A, Rompaey V, Maes LK, Heyning PH, Wuyts FL. Mal de debarquement syndrome: a systematic review. J Neurol. 2016;263(5):843-54.
9. Ortiz de Gortari AB. The Game Transfer Phenomena framework: investigating altered perceptions, automatic mental processes and behaviors induced by virtual immersion. Annu Rev Cyberther Telemed. 2016;14:9-15.

10. Ortiz de Gortari AB. Empirical study on Game Transfer Phenomena in a location-based augmented reality game. Telematics Inform. 2017;35(2):382-96.

11. Ortiz de Gortari AB. Game Transfer Phenomena: origin, development and contributions to the videogame research field. In: AttrillSmith A, Fullwood C, Kuss D, Keep M, editors. Oxford Handbook of Cyberpsychology. Oxford: Oxford University Press; 2019. p. 532-56.

12. Ortiz de Gortari AB, Aronsson K, Griffiths MD. Game Transfer Phenomena in video game playing: a qualitative interview study. Int J Cyber Behav Psychol Learn. 2011;1(3):15-33.

13. Ortiz de Gortari AB, Griffiths MD. Altered visual perception in Game Transfer Phenomena: an empirical self-report study. Int J Hum Comput Interact. 2014a;30(2):95-105.

14. Ortiz de Gortari AB, Griffiths MD. Auditory experiences in Game Transfer Phenomena: an empirical self-report study. Int J Cyber Behav Psychol Learn. 2014b;4(1):59-75.

15. Ortiz de Gortari AB, Griffiths MD. Automatic mental processes, automatic actions and behaviours in Game Transfer Phenomena: an empirical self-report study using online forum data. Int J Ment Heal Addict. 2014c;12(4):432-52.

16. Ortiz de Gortari AB, Griffiths MD. Game Transfer Phenomena and its associated factors: an exploratory empirical online survey study. Comput Hum Behav. 2015;51:195-202.

17.• Ortiz de Gortari AB, Griffiths MD. Prevalence and characteristics of Game Transfer Phenomena: a descriptive survey study. Int J Hum Comput Interact. 2016;32(6):470-80 This was the first published study that examined the prevalence and characteristics of Game Transfer Phenomena.

18. Ortiz de Gortari AB, Griffiths MD. Beyond the boundaries of the game: the interplay between in-game phenomena, structural characteristics of video games, and Game Transfer Phenomena. In: Gackenbach J, Bown J, editors. Boundaries of Self and Reality Online. San Diego: Academic Press; 2017. p. 97-121.

19. Ortiz de Gortari AB, Larøi F. Broadening the understanding of the psychopathology of gaming: the relation between involuntary behaviours, sensorial and cognitive intrusions with videogame content and Internet Gaming Disorder. J Behav Addict. 2018;7(1):24.

20.• Ortiz de Gortari AB, Oldfield B, Griffiths MD. An empirical examination of factors associated with Game Transfer Phenomena severity. Comput Hum Behav. 2016;64:274-84 This was the first study that examined the severity levels of GTP and its related predictor factors.

21. Ortiz de Gortari AB, Pontes H, Griffiths MD. The Game Transfer Phenomena Scale: an instrument for investigating the nonvolitional effects of video game playing. Cyberpsychol Behav Soc Netw. 2015;18(10):588-94.

22. Schimmenti A, Caretti V. Videoterminal dissociative trance: toward a psychodynamic understanding of problematic internet use. Clin Neuropsychiatry. 2017;14(1):64-72.

23. Slater M, Spanlang B, Sanchez-Vives MV, Blanke O. First person experience of body transfer in virtual reality. PLoS One. 2010;5(5): e10564.

24. Van Heugten-van der Kloet D, Cosgrave J, van Rheede J, Hicks S. Out-of-body experience in virtual reality induces acute dissociation. Psychol Conscious Theory Res Pract. 2018;5(4):346-57.

Publisher's Note Springer Nature remains neutral with regard to jurisdictional claims in published maps and institutional affiliations. 